\title{
Lithium Dendrite Growth Control Using Local Temperature Variation
}

Asghar Aryanfar ${ }^{1 *}$, Agustín J Colussi ${ }^{2}$ and Michael R. Hoffmann ${ }^{2}$

${ }^{1}$ Mechanical Engineering, ${ }^{2}$ Environmental Science and Engineering, California Institute of Technology, Pasadena, CA 91125, U.S.A.

\begin{abstract}
We have quantified lithium dendrite growth in an optically accessible symmetric Limetal cell, charged under imposed temperatures on the electrode surface. We have found that the dendrite length measure is reduced up to $43 \%$ upon increasing anodic temperature of about $50{ }^{\circ} \mathrm{C}$. We have deduced that imposing higher temperature on the electrode surface will augment the reduction rate relative to dendritic peaks and therefore lithium holes can draw near with the sharp deposited tips. We have addressed this mechanism via fundamentals of electrochemical transport.
\end{abstract}

\section{INTRODUCTION}

In recent years, wireless revolution and need for harnessing intermittent renewable energy sources, has created an exponential demand for long-lasting and high capacity energy storage devices with high-power delivery, such as batteries [1, 2]. Lithium metal, particularly, as a candidate for anode material with an ideal energy density of $3862 \mathrm{mAh} / \mathrm{g}$, could drastically address this demand. However, it has high propensity to grow dendrites during subsequent charging periods. The dendrites might eventually lead to short-circuit and initiate a current within the cell which causes overheating and ignition of the organic electrolyte solvents commonly used in such devices $[3,4]$.

The current reports on lithium dendrite have investigated the effect of charging method [5, 6] current density [7-9], electrode morphology[10-12], solvent and electrolyte chemical composition [13-15], and evolution time [16] on dendrite growth. Other methods include the use of powder electrodes [17] and adhesive polymers [18] and describing concentration variations $[19,20]$. Existing useful dendrite characterizations naturally involve simplifying assumptions such as one dimensional cell geometry that may have fallen short of capturing the comprehensive essentials of dendrite growth in realistic systems [8,21].

On the temperature effect, researchers have found that cycling at higher temperatures (from $50^{\circ} \mathrm{C}$ up to $40^{\circ} \mathrm{C}$ ) can, on average, cause more and more frequent short-circuiting events up to a factor of 2 [22]. Other results show that the increasing cell temperature enhances the ionic mobilities in favor of dendritic inception and growth [23]. [19, 24] reported that the higher temperatures extends ion depletion layer length which is in agreement reaction rates (probability of ionic reduction) direction correlation with temperature [25]. In contrast,[26] found that

\footnotetext{
*Corresponding Author: aryanfar@caltech.edu
} 
imposing higher temperatures reduces dendrite growth rate relatively to the electrode surface, and could results in more uniform deposition. Although all those approaches are helpful, it is apparent that further progress in tackling this crucial issue should accrue from a full understanding of the dynamics of dendrite growth on Li-metal electrodes.

Temperature, as an accessible physical parameter, has direct correlation with reduction rate during electrodeposition. However it's tuning for controlling dendrite growth rate and growing morphology has not yet been implemented. In this paper, we show experimental results that temperature variation across the cell from anode to cathode can restrain dendrites to a considerable amount.

\section{EXPERIMENTS}

In order to observe dendrites, we have used unique manually-made cells. The fabrication process has been explained in previous publication [5].

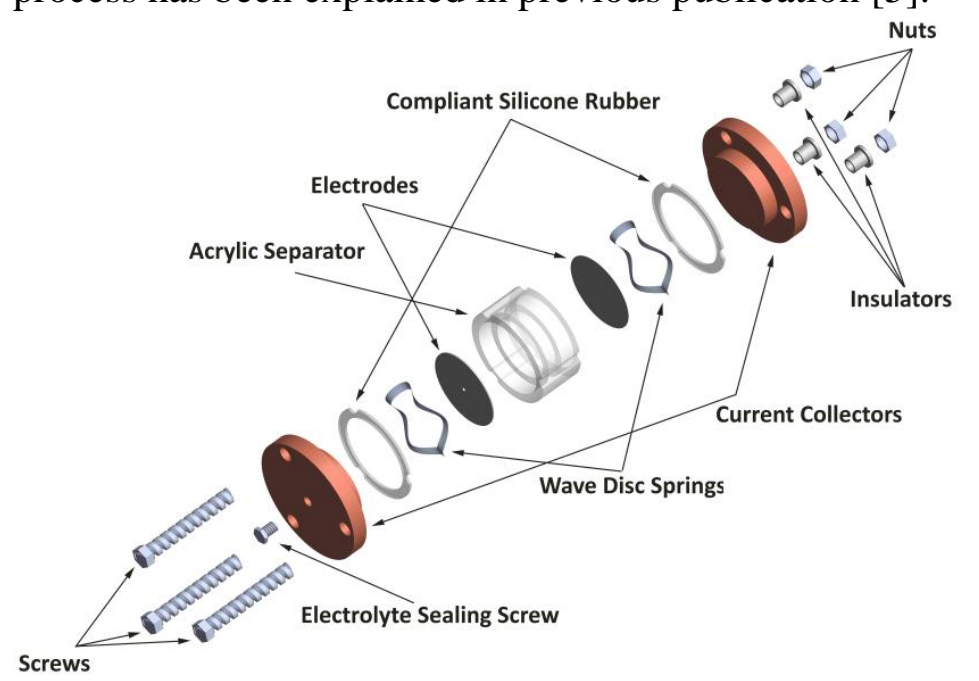

Fig. 1: Component-level view of the cell. The optical dendrite observation is possible from the transparent strip in acrylic separator.

Lithium foil (Aldrich, $99.9 \%$ on trace metal basis) $0.38 \mathrm{~mm}$ thick was punched and the oxide layers were scraped out via a sharp blade and DMC. The clean electrodes were flattened by being rolled via a glass tube. For each cell, 2 electrodes were intercalated in the separator. The components were sandwiched with insulated screws. For electrolyte, $1 \mathrm{M} \mathrm{LiClO}_{4}$ in $\mathrm{PC}$ was synthesized by stoichiometric mixing of $\mathrm{LiClO}_{4}$ (Aldrich, battery grade, $99.99 \%$ trace metal basis) that was dried for 24 hours in a vacuum oven at $100^{\circ} \mathrm{C}$ and was dissolved in PC (Aldrich, 99.7\% Anhydrous). The electrolyte was injected into each cell and the hole was plugged through a small screw lined with Teflon tape.

In order to impose the intended temperature gradient within the cells, we have submerged them up to anode level into a temperature-controlled oil bath (Fig. 2). Certainly, the temperature distribution in the cell medium starts to vary immediately from anode to cathode until it reaches 
the steady state regime. In the absence of dendrites, there is a steady heat flux across the entire cell from the higher to lower temperature regions and temperature varies linearly with respect to distance from anode. The experiments have been done in steady state regime inside argon-filled glovebox $\left(\mathrm{H}_{2} \mathrm{O}, \mathrm{O}_{2}<0.5 \mathrm{ppm}\right)$.

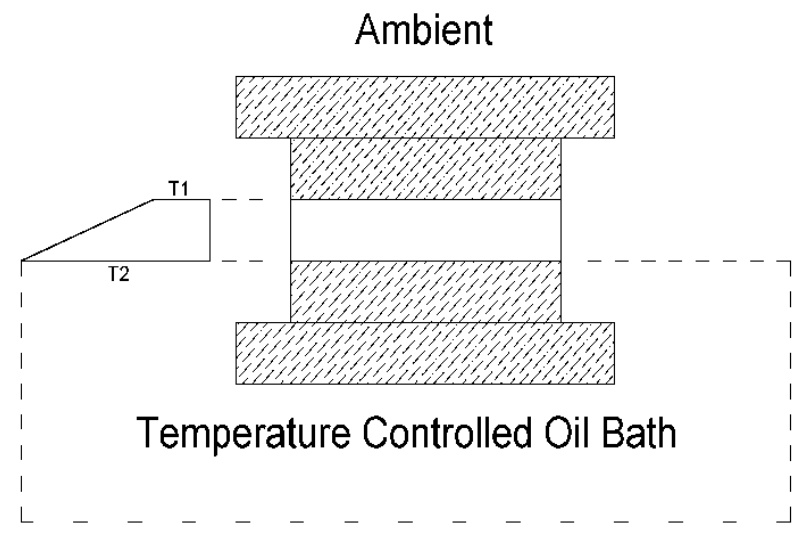

Fig. 2: Schematic diagram of set up for temperature gradient effect. The original temperature distribution before dendrite growth is demonstrated.

The cells were charged at the rate of $2 \mathrm{~mA} / \mathrm{cm}^{2}$ for 24 hours $(48 \mathrm{mAh})$ in ambient condition and two selected higher anodic (bath) temperatures using Bio-logic instruments (SP-50, VSP) and Neware battery tester (Shenzhen, China). After each experiment, the cells were disconnected and were rinsed with isopropanol. For each cell 3 high resolutions optical images were obtained from $120^{\circ}$ equiangular cell perimeter via Leica 205FA microscope. After integrating the images and projecting them into flat surface, 45 equidistance dendrite length measurements were been done on the lateral view of post-charge anode surface. The measure of dendrite length, $\bar{\lambda}$, is defined as the average of the measurements for each cell and is normalized to inter-electrode distance as follows:

$$
\bar{\lambda}=\frac{\sum_{i=1}^{N} \lambda_{i}}{L . N}
$$

where $N$ is number of measurements, $\lambda_{i}$ is individual dendrite length and $L$ is the interelectrode distance.

\section{DISCUSSION}

Dendrite growth relies on Brownian ionic movements and arbitrary bonding during ECD which naturally leads to non-deterministic microstructure formation in advancing electrode into the electrolyte. As $\mathrm{Li}^{+}$ions drift into the electrolyte from cathode to anode during battery recharge, the ultimate step that occurs in the double layer region in the vicinity of deposition sites is reduction to $\mathrm{Li}^{0}$. The key approach in our experiments is to alter the temperature within the cell domain such to tune the reduction rate in the regions close to electrode surface versus dendritic tips further from the electrode. Our kinetics dependent approach can be obtained indirectly from temperature-viscosity inverse relation as well (Walden's rule). 


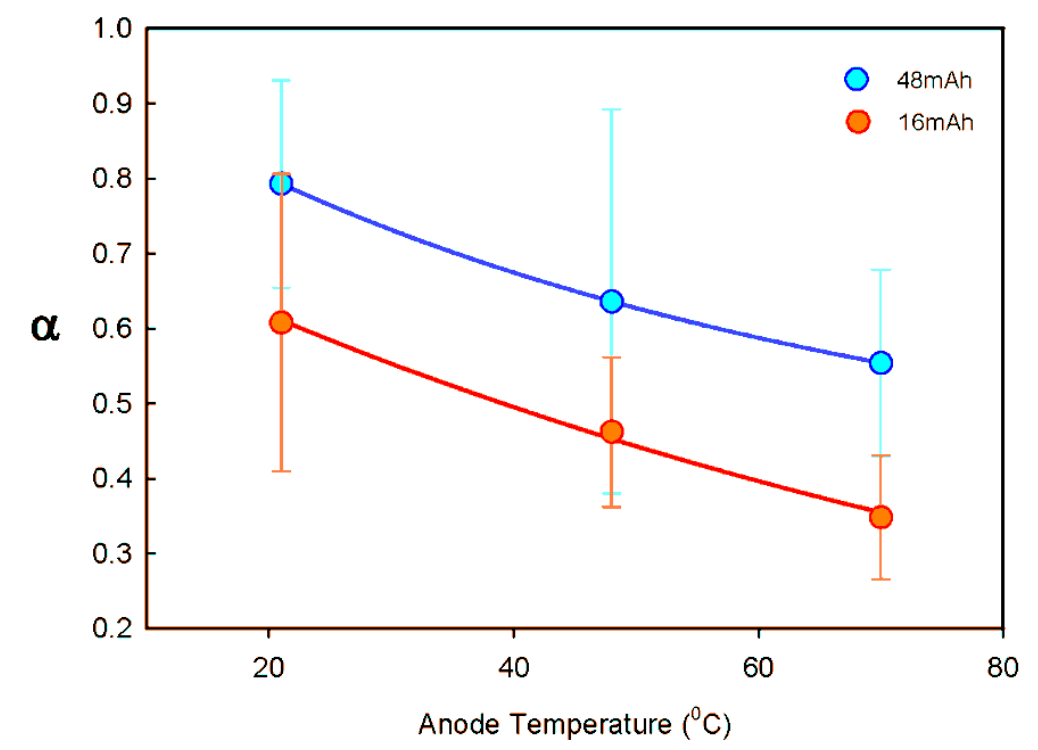

Fig. 3: Anodic temperature effect on dendrite length measure. The normalized average dendrite length can be reduced up to $42 \%$ by applying higher temperature up to $50^{\circ} \mathrm{C}$.

Our experimental results revealed that typically shorter dendrites were formed at higher imposed anodic temperatures with other cell parts in ambient condition (Fig. 3-a). In fact, imposing a higher temperature on the anode surface than ambient would, in average, favor deposition in those regions over dendrite tips since the reaction rate constant $(k)$ correlates directly with temperature by Arrhenius relation. On the other hand, the diffusion coefficient (D) also increases with temperature, which means that ions travel faster in inner layers to be reduced to $\mathrm{Li}^{0}[25]$. Thereby warmer less grown regions could possibly get closer to relatively colder dendritic tips. This trend is also consistent with previous studies [22, 26]. This fundamental adjustment can result in more uniform deposition and can inhibit growth runaway. We also note that the Fig. 3 curves are concave. We ascribe that applying much higher temperature could heat up the internal cell areas, enhance the deposition rate in those regions and lead to longer dendrites [22].

\section{CONCLUSIONS}

In this paper, we showed that temperature variations nearby electrode surface can alter the deposition kinetics effectively in favor of formation of more uniform morphology. We ascribed that imposing higher temperature nearby anode surface increases the reaction rate in regions closer to the anode surface (holes) relative to the other regions inside the cell, including the dendritic peaks.

\section{ACKNOWLEDGMENTS}

The authors acknowledge financial support from Bill and Melinda Gates Foundation Grant No. OPP1069500 on environmental sustainability and energy conservation.

\section{REFERENCES}


1. Tarascon, J.-M. and M. Armand, Issues and challenges facing rechargeable lithium batteries. Nature, 2001. 414(6861): p. 359-367.

2. Armand, M. and J.M. Tarascon, Building better batteries. Nature, 2008. 451(7179): p. 652-657.

3. Williard, N., et al., Lessons Learned from the 787 Dreamliner Issue on Lithium-Ion Battery Reliability. Energies, 2013. 6(9): p. 4682-4695.

4. $\mathrm{Xu}, \mathrm{K}$., Nonaqueous liquid electrolytes for lithium-based rechargeable batteries. Chemical Reviews-Columbus, 2004. 104(10): p. 4303-4418.

5. Aryanfar, A., et al., Dynamics of Lithium Dendrite Growth and Inhibition: Pulse Charging Experiments and Monte Carlo Calculations. The Journal of Physical Chemistry Letters, 2014: p. 1721-1726.

6. Mayers, M.Z., J.W. Kaminski, and T.F. Miller III, Suppression of Dendrite Formation via Pulse Charging in Rechargeable Lithium Metal Batteries. The Journal of Physical Chemistry C, 2012. 116(50): p. 26214-26221.

7. F. Orsini, A.D.P., B. Beaudoin, J.M. Tarascon, M. Trentin, N. Langenhuisen, E.D. Beer, P. Notten, In Situ Scanning Electron Microscopy (SEM) observation of interfaces with plastic lithium batteries. Journal of power sources, 1998. 76: p. 19-29.

8. Monroe, C. and J. Newman, Dendrite growth in lithium/polymer systems - A propagation model for liquid electrolytes under galvanostatic conditions. Journal of the Electrochemical Society, 2003. 150(10): p. A1377-A1384.

9. Nishida, T., et al., Optical observation of Li dendrite growth in ionic liquid. Electrochimica Acta, 2013.

10. Monroe, C. and J. Newman, The effect of interfacial deformation on electrodeposition kinetics. Journal of the Electrochemical Society, 2004. 151(6): p. A880-A886.

11. Aurbach, D., et al., A short review of failure mechanisms of lithium metal and lithiated graphite anodes in liquid electrolyte solutions. Solid State Ionics, 2002. 148(3): p. 405416.

12. Liu, X.H., et al., Lithium fiber growth on the anode in a nanowire lithium ion battery during charging. Applied Physics Letters, 2011. 98(18).

13. Crowther, O. and A.C. West, Effect of electrolyte composition on lithium dendrite growth. Journal of the Electrochemical Society, 2008. 155(11): p. A806-A811.

14. Schweikert, N., et al., Suppressed lithium dendrite growth in lithium batteries using ionic liquid electrolytes: Investigation by electrochemical impedance spectroscopy, scanning electron microscopy, and in situ Li-7 nuclear magnetic resonance spectroscopy. Journal of Power Sources, 2013. 228: p. 237-243.

15. Howlett, P.C., D.R. MacFarlane, and A.F. Hollenkamp, A sealed optical cell for the study of lithium-electrode electrolyte interfaces. Journal of Power Sources, 2003. 114(2): p. 277-284.

16. Rosso, M., et al., Onset of dendritic growth in lithium/polymer cells. Journal of Power Sources, 2001. 97-8: p. 804-806.

17. Seong, I.W., et al., The effects of current density and amount of discharge on dendrite formation in the lithium powder anode electrode. Journal of Power Sources, 2008. 178(2): p. 769-773.

18. Stone, G., et al., Resolution of the Modulus versus Adhesion Dilemma in Solid Polymer Electrolytes for Rechargeable Lithium Metal Batteries. Journal of The Electrochemical Society, 2012. 159(3): p. A222-A227. 
19. Brissot, C., et al., In situ concentration cartography in the neighborhood of dendrites growing in lithium/polymer-electrolyte/lithium cells. Journal of the Electrochemical Society, 1999. 146(12): p. 4393-4400.

20. Brissot, C., et al., Dendritic growth mechanisms in lithium/polymer cells. Journal of Power Sources, 1999. 81: p. 925-929.

21. Chazalviel, J.N., Electrochemical Aspects of the Generation of Ramified Metallic Electrodeposits. Physical Review A, 1990. 42(12): p. 7355-7367.

22. Park, H.E., C.H. Hong, and W.Y. Yoon, The effect of internal resistance on dendritic growth on lithium metal electrodes in the lithium secondary batteries. Journal of Power Sources, 2008. 178(2): p. 765-768.

23. Diggle, J., A. Despic, and J.M. Bockris, The mechanism of the dendritic electrocrystallization of zinc. Journal of The Electrochemical Society, 1969. 116(11): p. 1503-1514.

24. Brissot, C., et al., Concentration measurements in lithium/polymer-electrolyte/lithium cells during cycling. Journal of Power Sources, 2001. 94(2): p. 212-218.

25. Bard, A.J., and Larry R. Faulkner., Electrochemical methods: fundamentals and applications. 1980. 2 New York: Wiley, 1980.

26. Akolkar, R., Modeling dendrite growth during lithium electrodeposition at sub-ambient temperature. Journal of Power Sources, 2014. 246: p. 84-89. 